\title{
A NOTE ON RELATIVE AMENABILITY
}

\author{
PHILLIP WESOLEK
}

\begin{abstract}
P-E. Caprace and N. Monod isolate the class $\mathscr{X}$ of locally compact groups for which relatively amenable closed subgroups are amenable. It is unknown if $\mathscr{X}$ is closed under group extension. In this note, we exhibit a large, group extension stable subclass of $\mathscr{X}$, which suggests $\mathscr{X}$ is indeed closed under group extension. Along the way, we produce generalizations of the class of elementary groups and obtain information on groups outside $\mathscr{X}$.
\end{abstract}

\section{INTRODUCTION}

In 2, P-E. Caprace and N. Monod introduce the notion of relative amenability:

Definition 1.1. For a locally compact group $G$, a closed subgroup $H \leqslant G$ is relatively amenable if $H$ fixes a point in every non-empty convex compact $G$ space.

A convex compact $G$-space is a convex compact subset of a locally convex topological vector space such that the subset has a continuous affine $G$ action.

They go on to study the relationship between amenability and relative amenability. In particular, they isolate a large and interesting class of locally compact groups.

Definition 1.2. The class $\mathscr{X}$ is the collection of locally compact groups for which every relatively amenable closed subgroup is amenable.

Theorem 1.3 (Caprace-Monod, 2, Theorem 2]).

(a) $\mathscr{X}$ contains all discrete groups.

(b) $\mathscr{X}$ contains all groups amenable at infinity.

(c) $\mathscr{X}$ is closed under taking closed subgroups.

(d) $\mathscr{X}$ is closed under taking (finite) direct products.

(e) $\mathscr{X}$ is closed under taking adelic products.

(f) $\mathscr{X}$ is closed under taking directed unions of open subgroups.

Let $N \unlhd G$ be a closed normal subgroup of a locally compact group $G$.

(g) If $N$ is amenable, then $G \in \mathscr{X} \Longleftrightarrow G / N \in \mathscr{X}$.

(h) If $N$ is connected, then $G \in \mathscr{X} \Longleftrightarrow G / N \in \mathscr{X}$.

(i) If $N$ is open, then $G \in \mathscr{X} \Longleftrightarrow N \in \mathscr{X}$.

(j) If $N$ is discrete and $G / N \in \mathscr{X}$, then $G \in \mathscr{X}$.

(k) If $N$ is amenable at infinity and $G / N \in \mathscr{X}$, then $G \in \mathscr{X}$.

Date: June 2014.

2010 Mathematics Subject Classification. Primary 22D05.

Key words and phrases. Amenability, Relative Amenability, Elementary groups. 
Two questions concerning the class $\mathscr{X}$ arise:

Question 1.4 (Caprace-Monod).

(1) Is $\mathscr{X}$ stable under group extension?

(2) Are there locally compact groups outside of $\mathscr{X}$ ?

Our note contributes primarily to the study of the former. Indeed, let $\mathscr{Y}$ be the smallest collection of locally compact groups such that

(1) $\mathscr{Y}$ contains all compact groups, discrete groups, and connected groups,

(2) $\mathscr{Y}$ is closed under group extensions, and

(3) $\mathscr{Y}$ is closed under directed unions of open subgroups. That is to say, if $G=\bigcup_{i \in I} O_{i}$ where $\left\{O_{i}\right\}_{i \in I}$ is a directed system of open subgroups of $G$ such that $O_{i} \in \mathscr{Y}$ for each $i$, then $G \in \mathscr{Y}$.

We prove the following:

Theorem 1.5. The class $\mathscr{Y}$ is contained in $\mathscr{X}$ and enjoys the following additional permanence properties:

(a) $\mathscr{Y}$ is closed under taking closed subgroups.

(b) $\mathscr{Y}$ is closed under taking quotients by closed normal subgroups.

This provides evidence the class $\mathscr{X}$ may be stable under group extensions. Furthermore, it gives information on how groups $G \notin \mathscr{X}$ look like; see Remark 4.4 below.

Acknowledgments. The author would like to thank Pierre-Emmanuel Caprace for his many helpful suggestions and for supporting a week research stay at the Université catholique de Louvain during which this note was partially developed. This note was further developed during a visit to the Fields Institute; the author thanks the Institute for its hospitality.

\section{Generalities on locally compact Groups}

All groups are taken to be Hausdorff topological groups. We abbreviate "locally compact" by "l.c.", "totally disconnected" by "t.d.", and "second countable" by "s.c.". We write $H \leqslant_{o} G$ to indicate $H$ is an open subgroup of $G$. We denote by $\mathcal{U}(G)$ the collection of compact open subgroups of $G$.

As its statement is not the obvious generalization, we recall the first isomorphism theorem for locally compact groups (see [4, (5.33)]):

Let $G$ be a locally group, $A \leqslant G$ be closed, and $H \unlhd G$ be closed. If $A$ is $\sigma$-compact and $A H$ is closed, then $A H / H \simeq A / A \cap H$ as topological groups.

Locally compact $\sigma$-compact groups are close to being second countable.

Theorem 2.1 (Kakutani-Kodaira, see [4, (8.7)]). If $G$ is a $\sigma$-compact t.d.l.c. group, then there is a compact $K \unlhd G$ such that $G / K$ is metrizable, hence second countable.

A t.d.l.c. group $G$ is said to be a small invariant neighborhood group, denoted SIN, if $G$ admits a basis at 1 of compact open normal subgroups. These groups when compactly generated admit a useful characterization. 
Theorem 2.2 (Caprace-Monod, [1, Corollary 4.1]). A compactly generated t.d.l.c. group is SIN if and only if it is residually discrete.

The discrete residual of a t.d.l.c. group $G$, denoted $\operatorname{Res}(G)$, is the intersection of all open normal subgroups. When $G$ is compactly generated, Theorem 2.2 implies $G / \operatorname{Res}(G)$ is a SIN group.

Groups "built by hand" from profinite and discrete groups often appear when studying t.d.l.c. groups. The class of elementary groups captures the intuitive idea of such groups.

Definition 2.3. The class of elementary groups is the smallest class $\mathscr{E}$ of t.d.l.c.s.c. groups such that

(1) $\mathscr{E}$ contains all second countable profinite groups and countable discrete groups.

(2) $\mathscr{E}$ is closed under taking group extensions of second countable profinite or countable discrete groups. I.e. if $G$ is a t.d.l.c.s.c. group and $H \unlhd G$ is a closed normal subgroup with $H \in \mathscr{E}$ and $G / H$ profinite or discrete, then $G \in \mathscr{E}$.

(3) If $G$ is a t.d.l.c.s.c. group and $G=\bigcup_{i \in \mathbb{N}} O_{i}$ where $\left(O_{i}\right)_{i \in \mathbb{N}}$ is an $\subseteq$ increasing sequence of open subgroups of $G$ with $O_{i} \in \mathscr{E}$ for each $i$, then $G \in \mathscr{E}$. We say $\mathscr{E}$ is closed under countable increasing unions.

The class $\mathscr{E}$ enjoys robust permanence properties, which supports the thesis $\mathscr{E}$ is exactly the groups "built by hand."

Theorem 2.4 ([7, Theorem 3.18, Theorem 5.7]). Eீ enjoys the following permanence properties:

(a) $\mathscr{E}$ is closed under group extension.

(b) If $G \in \mathscr{E}, H$ is a t.d.l.c.s.c. group, and $\psi: H \rightarrow G$ is a continuous, injective homomorphism, then $H \in \mathscr{E}$. In particular, $\mathscr{E}$ is closed under taking closed subgroups.

(c) $\mathscr{E}$ is closed under taking quotients by closed normal subgroups.

(d) If $G \in \mathscr{E}, H$ is a t.d.l.c.s.c. group, and $\psi: G \rightarrow H$ is a continuous, injective homomorphism with dense normal image, then $H \in \mathscr{E}$.

The class $\mathscr{E}$ admits a canonical rank function:

Definition 2.5. The decomposition rank $\xi: \mathscr{E} \rightarrow\left[1, \omega_{1}\right)$ is an ordinal-valued function satisfying the following properties:

(a) $\xi(G)=1$ if and only if $G \simeq\{1\}$;

(b) If $G \in \mathscr{E}$ is non-trivial and $G=\bigcup_{i \in \mathbb{N}} O_{i}$ with $\left(O_{i}\right)_{i \in \mathbb{N}}$ some $\subseteq$-increasing sequence of compactly generated open subgroups of $G$, then

$$
\xi(G)=\sup _{i \in \mathbb{N}} \xi\left(\operatorname{Res}\left(O_{i}\right)\right)+1 .
$$

By [7, Theorem 4.7] and [7, Lemma 4.10], such a function $\xi$ exists, is unique, and is equivalent to the decomposition rank as it is defined in 7 .

The decomposition rank has a useful permanence property.

Lemma 2.6 (5, Lemma 2.9]). Suppose $G$ is a t.d.l.c.s.c. group and $N$ is a closed cocompact normal subgroup of $G$. If $N \in \mathscr{E}$, then $G \in \mathscr{E}$ with $\xi(G)=\xi(N)$. 


\section{The ClAss $\mathscr{E}^{*}$}

We first argue $\mathscr{E}$ is a subclass of $\mathscr{X}$. To this end, we require a lemma due to Caprace and Monod.

Lemma 3.1 ([2, Lemma 13]). Suppose $G$ is a locally compact group, $H \unlhd G$ is closed, and $O \leqslant_{o} G$ contains $H$. If $O \in \mathscr{X}$ and $G / H \in \mathscr{X}$, then $G \in \mathscr{X}$.

Theorem 3.2. $\mathscr{E} \subseteq \mathscr{X}$.

Proof. We argue by induction on $\xi(H)$ for $H \in \mathscr{X}$. As the base case is obvious, suppose $\xi(H)=\beta+1$ and write $H=\bigcup_{i \in \mathbb{N}} O_{i}$ with $\left(O_{i}\right)_{i \in \mathbb{N}}$ an inclusion increasing sequence of compactly generated open subgroups of $H$.

Fixing $i \in \mathbb{N}$, the quotient $O_{i} / \operatorname{Res}\left(O_{i}\right)$ is a SIN group via Theorem 2.2. It is therefore compact-by-discrete, and Theorem 1.3 implies it is in $\mathscr{X}$. On the other hand, the definition of the decomposition rank gives that $\xi\left(\operatorname{Res}\left(O_{i}\right)\right) \leq \beta$, so taking a compact open subgroup $V \leq O_{i}$, the group $V \operatorname{Res}\left(O_{i}\right)$ is elementary with rank at most $\beta$ via Lemma 2.6. Applying the induction hypothesis, we deduce that $V \operatorname{Res}\left(O_{i}\right) \in \mathscr{X}$. Lemma 3.1 now ensures that $O_{i} \in \mathscr{X}$, and since $\mathscr{X}$ is closed under direct limits, we conclude that $H \in \mathscr{X}$, completing the induction.

We next relax the second countability assumption on $\mathscr{E}$ by introducing the following class:

Definition 3.3. The class $\mathscr{E}^{*}$ is the smallest collection of t.d.l.c. groups such that

(1) $\mathscr{E} *$ contains all profinite groups and discrete groups,

(2) $\mathscr{E}^{*}$ is closed under group extensions of profinite and discrete groups, and

(3) $\mathscr{E}^{*}$ is closed under directed unions of open subgroups.

There is an ordinal rank on $\mathscr{E} *$. For $G \in \mathscr{E}$, define

- $G \in \mathscr{E}_{0}^{*}$ if and only if $G$ is profinite or discrete.

- Suppose $\mathscr{E}_{\alpha}^{*}$ is defined. Put $G \in\left(\mathscr{E}^{*}\right)_{\alpha}^{e}$ if and only if there exists $N \unlhd G$ such that $N \in \mathscr{E}_{\alpha}^{*}$ and $G / N \in \mathscr{E}_{0}^{*}$. Put $G \in\left(\mathscr{E}^{*}\right)_{\alpha}^{l}$ if and only if $G=$ $\bigcup_{i \in I} H_{i}$ where $\left(H_{i}\right)_{i \in I}$ is an $\subseteq$-directed set of open subgroups of $G$ and $H_{i} \in \mathscr{E}_{\alpha}^{*}$ for each $i \in I$. Define $\mathscr{E}_{\alpha+1}^{*}:=\left(\mathscr{E}^{*}\right)_{\alpha}^{e} \cup\left(\mathscr{E}^{*}\right)_{\alpha}^{l}$.

- For $\lambda$ a limit ordinal, $\mathscr{E}_{\lambda}^{*}:=\bigcup_{\beta<\lambda} \mathscr{E}_{\beta}^{*}$.

Certainly, $\mathscr{E}^{*}=\bigcup_{\alpha \in \mathrm{ORD}} \mathscr{E}_{\alpha}^{*}$, so for $G \in \mathscr{E}^{*}$, we define

$$
\operatorname{rk}(G):=\min \left\{\alpha \in \mathrm{ORD} \mid G \in \mathscr{E}_{\alpha}^{*}\right\} .
$$

We call $\operatorname{rk}(G)$ the construction rank of $G$.

The construction rank has a number of nice properties.

Lemma 3.4. Let $G \in \mathscr{E}^{*}$. Then

(a) $\operatorname{rk}(G)$ is a successor ordinal when $\operatorname{rk}(G)$ is non-zero.

(b) If $G$ is compactly generated and has non-zero rank, then $\operatorname{rk}(G)$ is given by a group extension. I.e. if $\operatorname{rk}(G)=\beta+1$, there is $H \unlhd G$ such that $\operatorname{rk}(H)=\beta$ and $\operatorname{rk}(G / H)=0$.

(c) If $O \leqslant \leqslant_{o} G$, then $O \in \mathscr{E}^{*}$ and $\operatorname{rk}(O) \leqslant \operatorname{rk}(G)$.

Proof. These follow by transfinite induction on $\operatorname{rk}(G)$. 
Proposition 3.5. Let $G \in \mathscr{E}^{*}$ be $\sigma$-compact. If $K \unlhd G$ is compact with $G / K$ second countable, then $G / K \in \mathscr{E}$.

Proof. We induct on the construction rank of $G$. As the proposition is immediate if $\operatorname{rk}(G)=0$, suppose $\operatorname{rk}(G)=\alpha+1$.

Suppose $\operatorname{rk}(G)$ is given by a directed union, so there is $\left(O_{i}\right)_{i \in I}$ a directed system of open subgroups of $G$ such that $G=\bigcup_{i \in I} O_{i}$ with $\operatorname{rk}\left(O_{i}\right) \leqslant \alpha$ for each $i \in I$. Certainly,

$$
G / K=\bigcup_{i \in I} O_{i} K / K
$$

and we may find a countable subcover $\left(O_{i} K / K\right)_{i \in \mathbb{N}}$ since $G / K$ is Lindelöf. One checks this cover may be taken to be an increasing $\subseteq$-chain. Further, $O_{i} K / K \simeq$ $O_{i} /\left(O_{i} \cap K\right), \operatorname{rk}\left(O_{i}\right) \leqslant \alpha$, and $O_{i} \cap K$ is a compact normal subgroup of $O_{i}$ whose quotient is second countable. The induction hypothesis implies $O_{i} /\left(O_{i} \cap K\right) \in \mathscr{E}$, and as $G / K$ is the countable increasing union of $\left(O_{i} K / K\right)_{i \in \mathbb{N}}$, we conclude $G / K \in$ $\mathscr{E}$.

Suppose the construction rank of $G$ is given by a group extension; say $H \unlhd G$ is such that $\operatorname{rk}(H)=\alpha$ and $\operatorname{rk}(G / H)=0$. Since $H$ is $\sigma$-compact and $K$ compact, we have $H K / K \simeq H /(H \cap K)$, and $\operatorname{as} \operatorname{rk}(H)=\alpha$, the induction hypothesis implies $H /(H \cap K) \in \mathscr{E}$. On the other hand, $H K / H \unlhd G / H$ is closed, and the quotient

$$
(G / H) /(H K / H) \simeq G / H K \simeq(G / K) /(H K / K)
$$

is second countable and either discrete or compact. We conclude $G / K$ is a group extension of $G / H K$ by $H K / K$, hence $G / K \in \mathscr{E}$. This completes the induction, and we conclude the proposition.

Theorem 3.6. $\mathscr{E}^{*} \subseteq \mathscr{X}$.

Proof. Take $G \in \mathscr{E}^{*}$. Every locally compact group is the directed union of its open compactly generated subgroups. Since $\mathscr{X}$ is closed under directed unions, we may assume $G$ is compactly generated and therefore $\sigma$-compact. Applying Theorem 2.1 there is $K \unlhd G$ such that $G / K$ is second countable. Proposition 3.5 implies $G / K \in \mathscr{E}$, and by Theorem 3.2, we have that $G / K \in \mathscr{X}$. Since $K$ is compact, we deduce from Theorem 1.3 that $G \in \mathscr{X}$.

We conclude this section by noting three permanence properties of $\mathscr{E}^{*}$.

Theorem 3.7. $\mathscr{E}^{*}$ enjoys the following permanence properties:

(a) $\mathscr{E}^{*}$ is closed under group extension.

(b) If $G \in \mathscr{E}^{*}$, then every t.d.l.c. group admitting a continuous, injective homomorphism into $G$ also belongs to $\mathscr{E}^{*}$. In particular, $\mathscr{E}^{*}$ is closed under taking closed subgroups.

(c) $\mathscr{E}^{*}$ is closed under taking quotients by closed normal subgroups.

Proof. Claim (a) follows just in the case of $\mathscr{E}$; see the proof of [7, Proposition 3.5].

For (b), let $G \in \mathscr{E}^{*}$, let $H$ be a t.d.l.c. group, and let $\psi: H \rightarrow G$ be a continuous, injective homomorphism. Every locally compact group is the directed union of its compactly generated open subgroups. Since $\mathscr{E}^{*}$ is closed under directed unions, 
we may therefore assume $H$ is compactly generated. Therefore, $P:=\overline{\psi(H)}$ is also compactly generated.

Since $H$ is $\sigma$-compact, it has a compact normal subgroup $K$ such that $H / K$ is second countable; likewise, $P$ admits a compact normal subgroup $L \unlhd P$ so that $P / L$ is second countable. The subgroup $L \psi(K)$ is then a compact normal subgroup of $P$ so that $P / L \psi(K)$ is second countable. The group $H / \psi^{-1}(L) K$ is also second countable, and $\psi$ induces an injective, continuous homomorphism $\tilde{\psi}$ : $H / \psi^{-1}(L) K \rightarrow P / L \psi(K)$. Applying Proposition 3.5 we conclude $P / L \psi(K) \in \mathscr{E}$ and, via Theorem 2.4 $H / \psi^{-1}(L) K \in \mathscr{E}$.

On the other hand, we have an injection $\psi^{-1}(L) K \rightarrow \psi(K) L$ with the latter group residually discrete. The group $\psi^{-1}(L) K$ is then residually discrete, and it follows from Theorem 2.2 that $\psi^{-1}(L) K \in \mathscr{E}^{*}$. In view of part $(a)$, we deduce $H \in \mathscr{E}^{*}$ as desired.

For $(c)$, suppose $L \unlhd G$ is closed; as above, it suffices to consider the case that $G$ is compactly generated. In view of Proposition 3.5. there is $K \unlhd G$ compact such that $G / K \in \mathscr{E}$. The group $L K / K$ is a closed normal subgroup of $G / K$, so Theorem 2.4 implies

$$
(G / L) /(L K / L) \simeq G / L K \simeq(G / K) /(L K / K) \in \mathscr{E}
$$

On the other hand, $L K / L \simeq K /(K \cap L)$ with the latter group compact. We conclude $G / L$ is compact-by- $\mathscr{E}$ and, via $(a)$, belongs to $\mathscr{E}^{*}$.

\section{THE ClASs $\mathscr{Y}$}

In order to prove Theorem 1.5 we introduce the class $\mathscr{Y}^{*}$ consisting of those locally compact groups $G$ such that $G / G^{\circ} \in \mathscr{E}^{*}$ where $G^{\circ}$ is the connected component of the identity. By definition, we have that $\mathscr{E}^{*} \subseteq \mathscr{Y}$, hence $\mathscr{Y}^{*} \subseteq \mathscr{Y}$ since $\mathscr{Y}$ is stable under group extensions and contains all connected l.c. groups. We will eventually show that $\mathscr{Y}^{*}=\mathscr{Y}$. The proof of this relies on the following.

Proposition 4.1. The class $\mathscr{Y}^{*}$ enjoys the following permanence properties:

(a) $\mathscr{Y}^{*}$ is closed under directed unions of open subgroups.

(b) $\mathscr{Y}^{*}$ is closed under taking closed subgroups.

(c) $\mathscr{Y}^{*}$ is closed under taking group extensions.

(d) $\mathscr{Y}^{*}$ is closed under taking quotients by closed normal subgroups.

The proof of (c) requires the following subsidiary fact.

Lemma 4.2. Let $G$ be a $\sigma$-compact l.c. group with closed $L, P \unlhd G$ so that $L \geqslant G^{\circ}$. If $P \in \mathscr{E}^{*}$, then $\overline{P L} / L \in \mathscr{E}^{*}$.

Proof. Set $H:=\overline{P L} / L$ and let $\pi: P \rightarrow H$ be the restriction of the usual projection. The image of $\pi$ is a dense normal subgroup of $H$. Theorem 3.7 ensures $\mathscr{E}^{*}$ is closed under taking quotients, so we may assume $\pi$ is injective.

As $G$ is $\sigma$-compact, $P$ is $\sigma$-compact, and additionally, $H$ is $\sigma$ compact since $P$ has dense image in $H$. We may thus find $K \unlhd P$ and $R \unlhd H$ compact subgroups so that $P / K$ and $H / R$ are second countable. The subgroup $\pi(K)$ is then a compact normal subgroup of $H$ with $H / \pi(K) R$ second countable. 
The induced map $\tilde{\pi}: P / K \pi^{-1}(R) \rightarrow H / \pi(K) R$ is an injective, continuous homomorphism with dense normal image. Appealing to Proposition 3.5, we have $P / K \pi^{-1}(R) \in \mathscr{E}$. Theorem 2.4 thus implies $H / \pi(K) R \in \mathscr{E}$, and as $\mathscr{E} *$ is closed under group extensions, we conclude that $H \in \mathscr{E}^{*}$, verifying the lemma.

Proof of Proposition 4.1. (a) Let $G$ be the directed union of $\left(O_{i}\right)_{i \in I}$ with $O_{i} \leqslant{ }_{o} G$ and $O_{i} \in \mathscr{Y}^{*}$ for each $i \in I$. For each $i$, we have $G^{\circ}=O_{i}^{\circ} \leqslant O_{i}$ since $O_{i}$ is open. So $G / G^{\circ}=\bigcup_{i \in I} O_{i} / O_{i}^{\circ}$, and therefore, $G / G^{\circ} \in \mathscr{E}^{*}$.

(b) Given $H \leqslant G$, we have that $H / H \cap G^{\circ}$ embeds continuously into $G / G^{\circ}$. By Theorem 3.7, this implies $H / H \cap G^{\circ} \in \mathscr{E}^{*}$. On the other hand, $H \cap G^{\circ} \leqslant G^{\circ}$ with $G^{\circ}$ a connected locally compact group. By the solution to Hilbert's fifth problem, $G^{\circ}$ is pro-Lie. Let $K \unlhd G^{\circ}$ be compact such that $G^{\circ} / K$ is a Lie group. In view of Cartan's theorem, see [6, LG 5.42], $\left(H \cap G^{\circ}\right) K / K$ is a Lie group. Putting $J:=K \cap H \cap G^{\circ}$, we have

$$
H^{\circ} J / J=\left(H \cap G^{\circ} / J\right)^{\circ} \leqslant_{o}\left(H \cap G^{\circ}\right) / J,
$$

so $\left(H \cap G^{\circ}\right) / H^{\circ} J$ is discrete. Since we may find such $J$ inside arbitrarily small neighborhoods of 1 , the group $\left(H \cap G^{\circ}\right) / H^{\circ}$ is residually discrete. Via Theorem 2.2. we infer $\left(H \cap G^{\circ}\right) / H^{\circ}$ is a directed union of SIN groups and so in $\mathscr{E}^{*}$. Since $\mathscr{E}^{*}$ is closed under group extension, $H / H^{\circ} \in \mathscr{E}^{*}$, whereby $H \in \mathscr{Y}^{*}$ as desired.

(c) Let $H \unlhd G$ be such that $H$ and $G / H$ both belong to $\mathscr{Y}^{*}$. To show $G \in \mathscr{Y}^{*}$, we may reduce to $G$ compactly generated. Indeed, if $O \leqslant_{o} G$ is compactly generated, then

$$
H^{\circ}=(O \cap H)^{\circ} \leqslant O \cap H \unlhd O
$$

and $(O \cap H) / H^{\circ} \leqslant_{o} H / H^{\circ}$. We thus deduce that $(O \cap H) / H^{\circ} \in \mathscr{E}^{*}$, whereby $O \cap H \in \mathscr{Y}^{*}$. Similarly, $O /(O \cap H) \in \mathscr{Y}^{*}$. The group $O$ is thus a group extension of groups in $\mathscr{Y}^{*}$. Therefore, if the claim holds for all compactly generated groups, then $O \in \mathscr{Y}^{*}$, and since $\mathscr{Y}^{*}$ is closed under directed unions, $G \in \mathscr{Y}^{*}$.

Suppose $G$ is compactly generated, put $\tilde{G}:=G /\left(H \cap G^{\circ}\right)$, and let $\pi: G \rightarrow \tilde{G}$ be the usual projection. Since $H^{\circ} \leqslant H \cap G^{\circ}, \tilde{H}:=\pi(H)$ is a quotient of $H / H^{\circ}$ and, via Theorem 3.7, a member of $\mathscr{E}^{*}$. Setting $\tilde{G}^{\circ}:=\pi\left(G^{\circ}\right)$, we apply Lemma 4.2 to conclude $\overline{\tilde{H} \tilde{G}^{\circ}} / \tilde{G}^{\circ} \in \mathscr{E} *$. Since

$$
\overline{\tilde{H} \tilde{G}^{\circ}} / \tilde{G}^{\circ} \simeq \overline{H G^{\circ}} / G^{\circ},
$$

we indeed have that $\overline{H G^{\circ}} / G^{\circ} \in \mathscr{E}^{*}$.

The connected component of any locally compact group coincides with the intersection of all its open subgroups. This implies $\overline{H G^{\circ}} / H=(G / H)^{\circ}$, so

$$
\left(G / G^{\circ}\right) /\left(\overline{H G^{\circ}} / G^{\circ}\right) \simeq G / \overline{H G^{\circ}} \simeq(G / H) /\left(\overline{H G^{\circ}} / H\right) \in \mathscr{E}^{*} .
$$

We conclude $G / G^{\circ}$ is an extension of a group in $\mathscr{E}^{*}$ by another group in $\mathscr{E}^{*}$ and thus a member of $\mathscr{E}^{*}$. Therefore, $G \in \mathscr{Y}^{*}$.

(d) Let $G \in \mathscr{Y}^{*}$ and $N \unlhd G$. As noticed above, we have $\overline{N G^{\circ}} / N=(G / N)^{\circ}$. Therefore, in order to show that $G / N \in \mathscr{Y}^{*}$, it suffices to show that $G / \overline{G^{\circ} N} \in \mathscr{E}^{*}$. The latter is isomorphic to a quotient of $G / G^{\circ} \in \mathscr{E}^{*}$, so the desired conclusion follows from Theorem 3.7 
Corollary 4.3. $\mathscr{Y}=\mathscr{Y}^{*} \subseteq \mathscr{X}$.

Proof. We have already observed that $\mathscr{Y}^{*} \subseteq \mathscr{Y}$. Since $\mathscr{Y}^{*}$ is stable under group extensions and directed unions of open subgroups by Proposition 4.1 the reverse inclusion follows. Taking $G \in \mathscr{Y}^{*}$, we see $G / G^{\circ} \in \mathscr{E}^{*} \subseteq \mathscr{X}$ and, via Theorem 1.3, $G \in \mathscr{X}$.

Proof of Theorem 1.5 . We have $\mathscr{Y} \subseteq \mathscr{X}$ by Corollary 4.3 , The permanence properties of $\mathscr{Y}$ follow from Proposition 4.1 .

Remark 4.4. The results of this note give new information concerning locally compact groups $G \notin \mathscr{X}$. As remarked in [2], we may take $G \notin \mathscr{X}$ to be a compactly generated t.d.l.c. group. Applying Theorem 2.1, we have a compact $K \unlhd G$ such that $G / K$ is second countable. Theorem 1.3 implies $G / K$ must also lie outside of $\mathscr{X}$. We may thus take $G \notin \mathscr{X}$ to be a compactly generated t.d.l.c.s.c. group.

By [7, Theorem 7.8], a t.d.l.c.s.c. group $G$ admits a unique maximal closed normal elementary subgroup, denoted $\operatorname{Rad}_{\mathscr{E}}(G)$ and called the elementary radical of $G$. Suppose $G$ is a t.d.l.c.s.c. group outside of $\mathscr{X}$ and fix $U \in \mathcal{U}(G)$. By Theorem 3.2, $\operatorname{Rad}_{\mathscr{E}}(G) \in \mathscr{X}$, and since $U \operatorname{Rad}_{\mathscr{E}}(G) \in \mathscr{E}$, we further have $U \operatorname{Rad}_{\mathscr{E}}(G) \in \mathscr{X}$. In view of Lemma 3.1, it must be the case $G / \operatorname{Rad}_{\mathscr{E}}(G) \notin \mathscr{X}$. We may thus suppose $G \notin \mathscr{X}$ has trivial elementary radical, and via 7, Corollary 9.12], $G$ is $[A]$-semisimple. The definition and a discussion of $[A]$-semisimple groups may be found in $[3$. Here we merely recall that $[A]$-semisimple groups have a canonical action on a lattice and, in many cases, a non-trivial boolean algebra 3.

\section{REFERENCES}

[1] P.-E. Caprace and N. Monod, Decomposing locally compact groups into simple pieces. Math. Proc. Cambridge Philos. Soc. 150 (2011) (1), 97-128.

[2] P.-E. Caprace and N. Monod, Relative amenability. Groups Geom. Dyn. 8 (2014) (3), 747774.

[3] P.-E. Caprace, C. Reid, and G. Willis, Locally normal subgroups of totally disconnected groups. Part I: General theory. Arxiv:1304.5144 [math.GR].

[4] E. Hewitt and K. A. Ross, Abstract harmonic analysis. Vol. I, vol. 115 of Grundlehren der Mathematischen Wissenschaften [Fundamental Principles of Mathematical Sciences] Springer-Verlag, Berlin-New York1979, second edn. Structure of topological groups, integration theory, group representations.

[5] C. D. Reid and P. R. Wesolek, Dense normal subgroups and chief factors in locally compact groups. Arxiv:1601.07317 [math.GR].

[6] J.-P. Serre, Lie algebras and Lie groups, vol. 1500 of Lecture Notes in Mathematics SpringerVerlag, Berlin1992, second edn. 1964 lectures given at Harvard University.

[7] P. Wesolek, Elementary totally disconnected locally compact groups. Proc. Lond. Math. Soc. (3) 110 (2015) (6), 1387-1434.

MSCS University of Illinois at Chicago, 322 Science and Engineering Offices MC249, 851 S. Morgan St. Chicago, Il 60607-7045, USA

Current address: Université Catholique de Louvain, Institut de Recherche en Mathématiques et Physique (IRMP), Chemin du Cyclotron 2, Box L7.01.02, 1348 Louvain-la-Neuve, BelGIUM

E-mail address: phillip.wesolek@uclouvain.be 